\title{
27 FAMILY MEMBERS' VIEWS AND EXPERIENCES OF DISCUSSING PREFERENCES AND WISHES FOR END-OF- LIFE CARE
}

10.1136/bmjspcare-2011-000053.27

G Horne', J Seymour ${ }^{2}$, S Payne ${ }^{3}{ }^{1}$ Rowcroft Hospice/University of Nottingham, Nottingham, UK; ${ }^{2}$ Palliative and End of Life Studies/University of Nottingham, Nottingham, UK; ${ }^{3}$ International Observatory on End of life Care/Help the Hospices Chair in Hospice Studies/University of Lancaster, UK

Aim This study is part of a larger cross-sectional study exploring the views of people affected by lung cancer about their experiences of discussing preferences and wishes for end of life care.

Design and method 19 family members (16 women, 3 men) took part in single, joint and group interviews conducted between: 2006 and 2008. Participants were mainly from lower socio-economic classes in the north of England, UK.

Findings The main findings were that family members supported the person with lung cancer to 'carry on as normal', which included continuing routine activities, planning holidays and special celebrations. They experienced emotional upset following disclosure of the person's prognosis and expressed feelings of loneliness and isolation when their needs for information about the future were not fulfilled. They also expressed the fear of being seen as 'disloyal' by the person with lung cancer if they initiated the conversation about plans for end of life care. Family members supported the person with lung cancer in practical planning for the future such as planning funerals, wills and financial plans. They had their own needs for practical planning in relation to sourcing equipment and services for the future to support them in their caring role.

Conclusion Health and social care professionals conducting advance care planning (ACP) need to consider the needs of family members about discussing preferences and wishes for end of life care, which may be different to those living with cancer. ACP interventions should be developed to meet the needs of both patients and their family members. 